\title{
Trends in der Angsttherapie
}

\author{
Dirk Wedekind und Borwin Bandelow \\ Klinik für Psychiatrie und Psychotherapie der Georg-August-Universität Göttingen
}

psychoneuro 2005; 31 (10): 499-503

$\mathrm{B}$ ei der Behandlung von Angststörungen wird heute eine Kombination von Pharmakotherapie und Kognitiver Verhaltenstherapie empfohlen. Zur Pharmakotherapie wurden in klinischen Studien vor allem Antidepressiva und Benzodiazepine untersucht (3). Ziele neuer Entwicklungen in der Pharmakotherapie sind Wirksamkeit bei Panikstörung, Sozialer Angststörung und Generalisierter Angststörung möglichst mit unmittelbarem oder zumindest raschem Wirkungsbeginn, gleichzeitig sehr guter Verträglichkeit und fehlenden Toleranzund Absetzeffekten. Zudem ist eine Optimierung unter Hinsicht auf eine Anwendung bei komorbiden Störungen und bei älteren Menschen zu beachten.

\section{Grenzen der modernen Pharmakotherapie}

Moderne, serotonerg wirksame Antidepressiva wie zum Beispiel die SSRI zeigen eine deutliche Verzögerung im Einsetzen der Wirksamkeit. Dies liegt am ehesten an der Dauer der Desensitivierung der präsynaptischen, somatodendritischen serotonergen (5HT $1 \mathrm{~A}$ )-Rezeptoren (24).

Die Behandlung mit Benzodiazepinen ist eingeschränkt durch eine mögliche Abhängigkeits- und Toleranzentwicklung und eine Reihe unerwünschter Begleiteffekte wie u.a. Sedierung. Eine solche Toleranzentwicklung kann sogar mit molekularen Veränderungen in Nervenzellen und einer folgenden pharmakologischen Responsivität ähnlich wie bei

Bei der Behandlung von Angststörungen wird heute eine Kombination von Pharmakotherapie und Kognitiver Verhaltenstherapie empfohlen. Neue Entwicklungen zielen auf schnellere, breitere Wirkung bei verbesserter Verträglichkeit, möglichst auch bei multimorbiden Patienten. Bei den Benzodiazepinen werden z.B. speichellösliche Darreichungsformen entwickelt. Neuentwicklungen bei den Antidepressiva sind z.B. das enatiomerenreine Escitalopram, retardiertes Paroxetin und der Serotonin- und Noradrenalin-Wiederaufnahmehemmer Duloxetin. Neue Behandlungsstrategien sind u.a. die Augmentation mit atypischen Antipsychotika. In der Pipeline stehen außerdem selektive Serotonin-Rezeptorliganden, neue GABA-A-Liganden, Antikonvulsiva, Neuropeptide, Neurosteroide und Modulatoren der neuroendokrinen Stressreaktion.

unbehandelten Angstpatienten einhergehen (33). Benzodiazepine haben auch einen recht unspezifischen Wirkmechanismus, indem sie die Wirkung des zentralnervösen GABASystems verstärken, das an der Mehrzahl der neuronalen Funktionen des Gehirns beteiligt ist.

\section{Benzodiazepine}

Ein Trend in der Entwicklung anxiolytisch wirksamer, hochpotenter Benzodiazepine sind speichellösliche Darreichungsformen für einen rascheren Wirkungseintritt, wie das bereits vermarktete Lorazepam-Lyophilisat (Tavor-expidet ${ }^{\circledR}$ ) und das in Deutschland nicht erhältliche Clonazepam-Lyophilisat (22). Eine interessante, hier ebenfalls noch nicht vermarktete Neuentwicklung ist auch eine retardierte Form von Alprazolam (Alprazolam $\mathrm{XR}^{\circledR}$ ), die sich bei lediglich einmaliger täglicher Einnahme durch ein günstigeres Nebenwirkungsprofil auszeichnet (32). Das Risiko einer Toleranz- und Abhängigkeitsentwicklung ist bei den neuen Darreichungsformen aber nicht als günstiger zu bezeichnen.

\section{neues bei den SSRI}

Bei den selektiven SerotoninWiederaufnahmehemmern (SSRI) gibt es viele Bemühungen, Effektivität und Verträglichkeit zu verbessern. Auch Erkenntnisse aus der klinischen Anwendung mit diesen Substanzen etablieren sich zunehmend in der Routineversorgung, wie z.B. SSRI nach längerer Einnahme grundsätzlich auszuschleichen und nicht abrupt abzusetzen, da sich hierbei häufiger unerwünschte Effekte zeigen (46). Orale Kontrazeptiva und eine Hormonersatztherapie können höhere Plasmaspiegel bestimmter SSRI verursachen (30). Auch der weibliche Zyklus kann die Plasmaspiegel dieser Antidepressiva beeinflussen. Die Halbwertszeit nimmt für viele Pharmaka über den Zyklus ab; andere werden um die Zeit der Ovulation am effektivsten metabolisiert. Möglicherweise besteht somit in der follikulären Phase ein vermehrtes Risiko für Nebenwirkungen, während in der Lutealphase das Rückfall- und Verschlechterungsrisiko für eine Angststörung höher ist (30).

Eine der neueren Entwicklungen ist Escitalopram, ein Enantiomer des 
Citalopram. Noch vor der Einführung in Deutschland befindet sich eine retardierte Form des Paroxetins, Paroxetin CR (controlled release), das sich bei gleich guter Wirksamkeit durch deutlich weniger Übelkeit bei Patienten zu Beginn der Behandlung auszeichnet (39). Die klinische Erfahrung zeigt, dass Serotonin-Wiederaufnahmehemmer allein nicht bei allen Patienten mit einer Angststörung effektiv sind. Aktuelle Arbeiten konnten zeigen, dass nicht nur bei der Zwangsstörung eine Augmentation mit bestimmten atypischen Antipsychotika eine weitere Besserung oder sogar eine Remission bewirken kann. Fallberichte zur Effektivität einer solchen Augmentationstrategie bei Angststörungen liegen für Aripiprazol (52), Ziprasidon (6) und Olanzapin (5) wie auch erste Studien (3) vor.

\section{Selektive Serotonin- Rezeptorliganden}

Ein Trend ist in der Entwicklung von Pharmaka mit einem spezifischeren Effekt auf bestimmte Serotonin-5HT-Rezeptorsubtypen zu sehen, die mit der Pathogenese von Angst in Verbindung stehen. Selektive $5 \mathrm{HT}_{2}$ (bzw. $5 \mathrm{HT}_{2 \mathrm{C}}$ ) oder den $5 \mathrm{HT}_{1 \mathrm{~A}}$-Rezeptorliganden sind entwickelt worden. Buspiron ist ein selektiver, partieller Agonist am $5 \mathrm{HT}_{1 \mathrm{~A}^{-}}$ Rezeptor, der positive Resultate in der Behandlung der generalisierten Angststörung zeigte. Die $5 \mathrm{HT}_{1 \mathrm{~A}}$-Bindungsstellen werden hierbei heruntergeregelt (35) und so die serotonerge Aktivität indirekt verstärkt. Schweizer (37) beschrieb günstigere Verläufe für Patienten mit vorrangig psychischen Symptomen mit Buspiron, während somatische Symptome besser auf Benzodiazepine ansprachen. Der partielle $5 \mathrm{HT}_{1 \mathrm{~A}}$-Agonist Gepiron zeigte Teileffekte bei der Panikstörung (28) und das Buspironanalogon MM199 hatte eine gute anxiolytische Potenz (7). Der vollständige $5 \mathrm{HT}_{1 \mathrm{~A}}$-Agonist Flesinoxan führt aber bei der Panikstörung zu einer Verschlechterung (47).

Deramciclan und Agomelatin haben ein spezifisches $5 \mathrm{HT}_{2 \mathrm{C}}$-antagonistisches Profil und einen anxiolytischen Effekt, werden aber primär zur Behandlung der Depression un- tersucht. Eine zusätzliche $5 \mathrm{HT}_{2 \mathrm{C}}$-antagonistische Komponente bei Serotonin-Wiederaufnahmehemmern könnte das Problem anxiogener Effekte in der frühen Behandlungsphase reduzieren. Kandidat wäre Mirtazapin, dies erbrachte positive Resultate bei der Panikstörung. Der $5 \mathrm{HT}_{3}$-Antagonist Ondansetron konnte in bisherigen Studien keine konkreten Effekte zeigen, und schlüssige Ergebnisse für eine ähnliche Substanz, Zatosetron, stehen noch aus.

Anxiogene Effekte bei Panikpatienten sind mit einer Dysfunktion des $5 \mathrm{HT}_{1 \mathrm{D}}$-Rezeptors in Verbindung gebracht worden. Die Verabreichung des postsynaptischen $5-\mathrm{HT}_{1 \mathrm{D}}$-Rezeptoragonisten Sumatriptan (das in der Migränetherapie verwendet wird) führte bei Patienten im Vergleich zu Kontrollen zu signifikant mehr Angstsymptomen (1). Die Entwicklung eines spezifischen $5 \mathrm{HT}_{1 \mathrm{D}}-\mathrm{Re}$ zeptorantagonisten könnte somit eine gute Strategie sein. In verschiedenen Tiermodellen für Angst konnten anxiolytische Effekte ähnlich wie mit Diazepam für die selektiven $5 \mathrm{HT}_{1 \mathrm{~B}}$-Liganden CP94253 und SB216641 gezeigt werden (44).

\section{Inositol}

Das Glukoseisomer Inositol ist Bestandteil vieler Nahrungsmittel. Inositolphosphat dient als Secondmessenger für einige Serotonin-Rezeptortypen, aber auch für noradrenerge Rezeptoren. Zwei klinische Studien konnten eine gute Wirksamkeit von Inositol bei der Panikstörung zeigen $(4,26)$.

\section{Neue GABA-A-Liganden}

GABA-Liganden wie Benzodiazepine haben gute anxiolytische Effekte, sind aber problematisch wegen der möglichen Abhängigkeitsentstehung und Absetzproblemen. Eine GABA-Dysfunktion ist eine der etablierten Theorien zur Pathogenese von Angststörungen. Man geht von einer Verschiebung des Benzodiazepin-Rezeptorkomplexes in die invers agonistische Richtung aus bzw. von einer Defizienz agonistischer, anxiolytischer Liganden (24). Auch konnte bei Angstpatienten in angstregulierenden Hirnstrukturen eine reduzierte Anzahl von Benzodiazepinrezeptoren nachgewiesen werden (33). Die angstlösenden Effekte werden über die $\alpha 2$ - und/ oder $\alpha 3$-Untereinheit des GABA-ARezeptors vermittelt, während sedierende Effekte $\alpha 1$ vermittelt sind (2). Nichtselektive, parzielle GABAA-Agonisten, wie Pagoclon, haben Teileffekte bei der Panikstörung ohne viele der bekannten unerwünschten Begleiteffekte und $\mathrm{Ab}$ setzschwierigkeiten (34). Bretazenil, Imidazenil, S-Desmethylzopiclon, FG8205, NS2710 und RWJ-51204 sind weitere Kandidaten aus dieser Gruppe. Selektive GABA-A-Agonisten, die an der $\alpha 2$ - und/oder $\alpha 3$-Untereinheit binden, werden untersucht (2).

\section{- Antikonvulsiva}

Gabapentin oder Pregabalin sind Liganden an der $\alpha$-2-Delta-Untereinheit spannungsabhängiger Kalziumkanäle (50). Eine kontrollierte Studie zeigte positive Ergebnisse bei schweren Panikstörungen für Gabapentin (27), während Pregabalin erfolgreich bei der Generalisiertenund Sozialen Angststörung untersucht wurde. Ergebnisse einer Studie bei Panikstörung stehen noch aus. Gabapentin eignet sich möglicherweise auch gut zur Augmentation bei ungenügendem Effekt von Serotonin-Wiederaufnahmehemmern bei der Sozialen Angststörung. Bei zuvor therapierefraktären Patienten mit einer Panikstörung konnten in einer Fallstudie Erfolge durch das Antikonvulsivum Tiagabin gezeigt werden. Auch Levetiracetam hatte in einer offenen, nicht kontrollierten Untersuchung deutliche anxiolytische Effekte bei Patienten mit einer Sozialen Angststörung (41). Interessanterweise konnte im Tierversuch gezeigt werden, dass die anxiolytische Potenz von Levetiracetam vom basalen Stressniveau abhängig ist (18).

\section{Glutamatmodulatoren}

Glutamat ist der am weitesten verbreitete exzitatorische Transmitter im Gehirn. Es gibt acht so genannte metabotrope Glutamatrezeptoren (mGLU1-8), die die glutamaterge Neurotransmission G-Pro- 
tein-gekoppelt, indirekt inhibitorisch oder exzitatorisch modulieren (11). Anxiolytisch wirksame Glutamatmodulatoren sollten entweder postsynaptisch antagonistische (wie LY-326325) oder präsynaptisch agonistische Eigenschaften haben. Tierdaten zeigen tatsächlich anxiolytische Effekte, insbesondere bei mGlu1 (14), mGlu2/3 (36) und mGlu5-Rezeptorantagonisten. Humanstudien (anxiolytische bei $\mathrm{CO} 2$ induzierten Panikattacken) (36) stehen noch am Anfang, aber aufgrund der Benzodiazepinen vergleichbaren, rasch einsetzenden Wirkung bei einem günstigen Nebenwirkungsprofil werden selektive Glutamatmodulatoren möglicherweise in der Zukunft eine wichtige Rolle spielen.

\section{Neuropeptide}

Von den Neuropeptiden, die im Besonderen bei Panik eine Rolle spielen, ist Cholecystokinin am besten untersucht. Das Tetrapeptid (CCK-4) und das synthethische Pentapeptid Pentagastrin können bei Gesunden und in bedeutend geringeren Dosen bei Menschen mit einer Panikstörung Panikattacken hervorrufen. CCK-4-Bindungsstellen finden sich besonders in limbischen Strukturen wie der Amygdala und dem Hippocampus sowie dem noradrenergen Kernzentrum, dem Locus coeruleus. Da CCK-4 in Provokationstests nicht nur Angstgefühle, sondern auch körperliche Angstsymptome ohne deutliche psychische Angst verursacht, könnte dies auf den Einfluss auf unterschiedliche Anteile des zentralnervösen „Angstnetzwerks“ hinweisen. CCK-4- (bzw. Neurokinin-3)-Rezeptorantagonisten konnten in den bisherigen Untersuchungen jedoch noch keine wesentlichen Effekte in der Behandlung, z.B. der Panikstörung zeigen (16), wenngleich diese aber durch CCK-4 induzierte Panikattacken unterdrücken konnten (45). Substanz P, ein Neurokinin-1-Rezeptor(NK-1)-Agonist, spielt eine wichtige Rolle bei Angst und Panik ähnlichem Verhalten und ist häufig in noradrenergen und serotonergen Zellen kolokalisiert (29). Untersuchungen mit Antagonisten wie MK869 bei Angst im Rahmen einer Depression zeigten gute Ergebnisse (15) und Stu- dien bei Panikstörung werden unternommen. Im Tierversuch wurde ein anxiolytischer Effekt durch den selektiven NK-1-Antagonisten NKP608 wiederholt demonstriert (48).

Neuropeptid-Y besteht aus 36 Aminosäuren. Es reguliert die zentralnervöse noradrenerge Aktivität und hat eine hohe Dichte von Bindungsstellen im Hypothalamus. Obwohl die meisten noradrenerg wirksamen Antidepressiva keine gute Wirksamkeit bei Angststörungen zeigten, gibt es doch Anhalt für die Effektivität des selektiven Noradrenalin-Wiederaufnahmehemmers Reboxetin (49). Ein Neuropeptid-YLigand könnte daher eine geeignete Substanz sein. Klinische Studien und eine oral verabreichbare synthetische Substanz stehen noch aus.

Limbische Sigmarezeptoren haben eine neuromodulatorische Funktion auf noradrenerge- und auch serotonerge Zellkernareale. Einige Substanzen befinden sich aktuell in der klinischen Erprobung. Offensichtlich zeichnen sich Sigmarezeptorliganden durch ein sehr günstiges Nebenwirkungsprofil aus, aussagekräftige Ergebnisse fehlen aber bisher.

\section{Neurosteroide und Modula- toren der neuroendokrinen Stressreaktion}

Viele psychiatrische Störungen zeigen eine anhaltende Hyperaktivität der Hypothalamus-Hypophysen-Nebennierenrinden(HPA)Achse und einer Hypersekretion von Kortisol. Hierzu zählen auch prinzipiell die Angststörungen. Modulatoren der neuroendokrinen Stressreaktion sind zuletzt intensiv beforscht worden. Der Steroid-Synthese-Blocker Ketoconazol zeigte bei depressiven Patienten mit einem Hyperkortisolismus zum Beispiel eine Plazebo überlegene Wirkung (51). Wie auch zum Glukokortikoid-Rezeptorantagonisten Mifepriston liegen jedoch noch keine Studien bei Angststörungen vor (23). Auch Metyrapon, ein weiterer Glukokortikoid-Synthesehemmer, erwies sich in Tiermodellen zur Depression (9) und in einer kleineren kontrollierten Depressionsstudie als effektiv.
Corticotropin-Releasing-Hormon (CRH) erzeugt verschiedene Symptome von Stress, Angst und Panik im zentralen Nervensystem. Die Modulierung der CRH-Aktivität ist Ziel der pharmakologischen Forschung bei Angststörungen. CRHAntagonisten können nach intraventrikulärer Applikation im Tierversuch Angst- und Stresssymptome vermindern (20). Antagonisten am CRH- oder Glukokortikoid-Rezeptor stellen für klinische Untersuchungen möglicherweise einen wesentlichen Fortschritt dar. Das gilt gleichermaßen für Agonisten am Rezeptor für atriales natriuretisches Peptid (ANP), das die Funktion der Hypothalamus-Hypophysen-Nebennieren-Achse an verschiedenen Stellen moduliert. Im Tierversuch hat ANP anxioytische Effekte und zuvor verabreichte ANP-Agonisten können bei Panikpatienten wie auch bei Gesunden Panikattacken durch eine CCK-4 Provokation unterdrücken (40).

Am weitesten fortgeschritten sind Studien mit CRH-1-Rezeptorantagonisten. Bei speziellen Rattenstämmen konnten sowohl Hyperkortisolismus als auch ängstliches Verhalten reduziert werden $(12,13)$. Eine offene Studie mit einem CRH-1 Antagonisten konnte bei Patienten mit Depression Angst- und Depressionssymptome bessern (10). Ein interessantes Konzept wäre die Kombination eines CRH-1-Antagonisten mit einem SSRI, um dessen Wirklatenz zu verkürzen.

Vasopressin ist ebenfalls ein relevanter Modulator der neuroendokrinen Stressreaktion und wahrscheinlich an der Entstehung bestimmter assoziierter Psychopathologien beteiligt. SSR149415 ist ein selektiver Vasopressin V(1b) Rezeptorantagonist, der im Tierexperiment sowohl antidepressive wie auch anxiolytische Effekte in verschiedenen Tiermodellen zur Generalisierten Angststörung zeigte (38). Klinische Untersuchungen sind in Planung.

Progesteron hat gewisse anxiolytische, aber auch dysphorisierende Effekte (8). Diese Wirkung wird durch einen modulierenden Einfluss auf GABA-Rezeptoren bedingt (21). 
Es besteht daher ein großes Interesse an Studien von Progesteronähnlichen Substanzen, z.B. Allopregnanolon, die eine gute anxiolytische Potenz aufweisen (194), und anderer neuroaktiver Steroide (31).

\section{Fazit}

Die z.Zt. absehbaren, wichtigsten Entwicklungen für die Zukunft anxiolytischer Pharmakotherapie sind Neuropeptid- und Neurosteroid-Rezeptor-Liganden, spezifische Modulatoren am GABA-A-BenzodiazepinRezeptorkomplex und Modulatoren des glutamatergen sowie des serotonergen Systems.

\section{Trends in the treatment of Anxiety Disorders}

For the treatment of anxiety disorders, a combination of pharmacotherapy and cognitive-behavioural measures is recommended. New developments are aimed at achieving a more rapid and broader effect, together with improved tolerability, as far as possible also in multimorbid patients. Among the benzodiazepines, saliva.soluble forms are being developed. New developments among the antidepressants, for example, include the pure S-enantiomer, escitalopram, slow-release paroxetine, and the serotonin and norepinephrine re-uptake inhibitor, duloxetine. New treatment strategies include augmentation with atypical antipsychotics. Furthermore, selective serotonin receptor ligands, new GABAA ligands, anticonvulsive drugs, neuropeptides, neurosteroids and modulators of the neuroendocrine stress reaction are all in the pipeline.

\footnotetext{
Literatur

1. Amital D, Fostick L, Sasson Y, Kindler S, Amital H, Zohar J. Anxiogenic effects of Sumatriptan in panic disorder: a double-blind, placebo controlled study. Eur Neropsychopharmacol 2005; 15 (3): 279-283

2. Atack JR. Anxioselective compounds acting at the $G A B A(A)$ receptor benzodiazepine binding site. Curr Drug Targets CNS Neurol Disord 2003; 2 (4): 213-232 3. Bandelow B, Zohar J, Hollander E et al. WFSBP Task force on treatment guidelines for the pharmacological treatment of anxiety-obsessive compulsive disorders and posttraumatic stress disorder. World J Biol Psychiatry 2002; 3: 171-199

4. Benjamin J, Levine J, Fux M, Aviv A, Levy D, Belmaker RH. Double-blind, placebo-con-
}

trolled, crossover trial of inositol treatment for panic disorder. Am J Psychiatry 1995; 152 (7):1084-6

5. Chao IL. Olanzapin augmentation in panic disorder: a case report. Pharmacopsychiatry 2004; 37 (5): 239-240

6. Crane DL. Ziprasidone as an augmenting agent in the treatment of anxietyspectrum disorders. CNS Spectr 2005; 10 (3): 176-179

7. Deren-Wesolek A, Tatarczynska E, Chojnacka-Wojcik E. The novel buspirone analogue, 8-(4-(2-(1,2,3,4-tetrahydroisoquinolinyl)(butyl)-8-azaspiro (4.5) decane7,9-dione, with aniolytic-like and antidepressant-like effects in rats. J Psychopharmacol 1998; 12 (4): 380-384

8. Halbreich U. Premenstrual dysphoric disorders: a diversified cluster of vulnerability traits to depression. Acta Psychiatr Scand 1997; 95 (3): 169-176

9. Healy DG, Harkin A, Cryan JF et al. Metyrapone displays antidepressant-like properties in preclinical paradigms. Psychopharmacology (Berl) 1999; 145 (3): 303-308

10 . Holsboer $F$. The rationale for corticotropin-releasing hormone receptor (CRH-R) antagonists to treat depression and anxiety. J Psychiatr Res 1999; 33: 181-214

11. Javitt DC. Glutamate as a therapeutic target in psychiatric disorders. Mol Psychiatry 2004; 9 (11): 984-997

12. Keck ME, Holsboer F. Hyperactivity of $\mathrm{CRH}$ neuronal circuits as a target for therapeutic interventions in affective disorders. Peptides 2001; 22 (5): 835-844

13. Keck ME, Welt T, Muller MB et al. The high-affinity non-peptide $\mathrm{CRH} 1$ receptor antagonist R121919 attenuates stressinduced alterations in plasma oxytocin, prolactin, and testosterone secretion in rats. Pharmacopsychiatry 2003; 36 (1): 27-31 14. Klodzynska A, Tatarczynska E, Stachowicz K, Chojnacka-Wojcik E. The anxiolyticlike activity of AIDA (1-aminoindan-1,5-dicarboxolic acid), an mGlu 1 receptor antagonist. J Physiol Pharmacol 2004; 55: $113-$ 126

15. Kramer MS, Cutler N, Feighner ] et al. Distinct mechanism for antidepressant activity by blockade of central substance $P$ receptors. Science 1998; 281 (5383): 16401645

16. Kronenberg G, Berger P, Tauber RF, Bandelow B, Henkel V, Heuser I. Randomized, double-blind study of SR142801 (Osanetant). A novel neurokinin-3 (NK3) receptor antagonist in panic disorder with pre- and posttreatment cholecystokinin tetrapeptide (CCK-4) challenges. Pharmacopsychiatry 2005; 38 (1): 24-29

17. Kuhl H.: Sexualhormone und Psyche. Stuttgart, New York: Thieme, 2002

18. Lamberty Y, Falter U, Gower AJ, Klitgaard $\mathrm{H}$. Anxiolytic profile of the antiepileptic drug levetiracetam in the Vogel conflict test in the rat. Eur J Pharmacol 2003; 469 (13): 97-102

19. Le Melledo JM, Baker G. Role of progesterone and other neuroactive steroids in anxiety disorders. Expert Rev Neurother 2004; 4(5): 851-860

20. Liebsch F, Landgraf R, Engelmann $M$ et al. Differential behavioural effects of chronic infusion of $\mathrm{CRH} 1$ and $\mathrm{CRH} 2$ receptor antisense oligonucleotides into the rat brain. J Psychiatry Res 1999; 33: 153-163

21. Majewska MD. Neurosteroids: endogenous bimodal modulators of the GABA-A receptor. Mechanisms of action and physiological significance. Prog Neurobiol 1992; 38 (4): 379-395

22. Moroz G. High-potency benzodiazepines: recent clinical results. J Clin Psychiatry 2004; 65 (suppl. 5): 13-18

23. Murphy BE, Filipini D, Ghadirian AM. Possible use of glucocorticoid receptor antagonists in the treatment of major depression: preliminary results using RU 486. J Psychiatry Neurosci 1993; 18 (5): 209-213

24. Nutt DJ, Forshall S, Bell C et al. Mechanisms of action of selective serotonin reuptake inhibitors in the treatment of psychiatric disorders. Eur Neuropsychopharmacol 1999; 9 (suppl. 3): 81-86

25. O'Dwyer AM, Lightman SL, Marks MN et al. Teatment of amjor depression with metyrapone and hydrocortisone. J Affect Disord 1995; 33 (2): 123-128

26. Palatnik A, Frolov K, Fux M, Benjamin J. Double-blind, controlled crossover trial of inositol versus fluvoxamine for the treatment of panic disorder. I Clin Psychopharmacol 2001; 21 (3): 335-9

27. Pande AC, Pollack MH, Crockatt ] et al. Placebo-controlled of gabapentin treatment of panic disorder. J Clin Psychopharmacol 2000; 20: 467-471

28. Pecknold JC, Luthe L, Scott Fleury MH et al. Gepirone and the treatment of panic disorder: an open study. J Clin Psychopharmacol 1993; 13: 145-149

29. Pelletier G, Steinbusch HW, Verhofstad AA. Immunoreactive substance $P$ and serotonin present in the same dense-core vesicles. Nature 1981; 293 (5827): 71-72

30. Pigott TA. Gender differences in the epidemiology and treatment of anxiety disorders. J Clin Psychiatry 1999; 60 (suppl 18): 4-15

31. Reddy DS. Pharmacology of endogenous neuroactive steroids. Crit. Rev. Neurobiol 2003; 15, 197-234

32. Rickels K. Alprazolam extended-release in panic disorder. Expert Opin Pharmacother 2004; 5 (7): 1599-1611

33. Roy-Byrne PP (2005) The GABA-benzodiazepine receptor complex: structure, function and role in anxiety. I Clin Psychiatry 66 (suppl.2): 14-20

34. Sandford J], Forshall S, Bell C et al. Crossover trial of pagoclone and placebo in patients with DSM-IV panic disorder. J Psychopharmacol 2001; 15 (3): 205-208

35. Sargent PA, Kjaer KH, Bench Cl et al. Brain serotonin $1 \mathrm{~A}$ receptor binding measured by positron emission tomography with (11C)WAY-100635: effects of depression and antidepresan treatment. Arch Gen Psychiatry 2000; 57 (2): 174-180

36. Schoepp DD, Wright RA, Levine LR, Gaydos B, Potter WZ. LY354740, an mGlu2/3 receptor agonist as a novel approach to treat anxiety/stress. Stress 2003; 6(3): 189-197 37. Schweizer E. Generalized anxiety disorder. Longitudinal course and pharmacologic 
treatment. Psychiatr Clin North Am 1995; 18 (4): 843-857

38. Serradeil-Le Gal C, Wagnon J 3rd, Tonnerre B, Roux R, Garcia G, Griebel G, Aulombard A. An overview of SSR149415, a selective nonpeptide vasopressin $\mathrm{V}(1 \mathrm{~b})$ receptor antagonist. CNS Drug Reviews 2005; 11: 53-68

39. Sheehan DV, Burnham DB, lyengar MK, Perera P; Paxil CR Panic Disorder Study Group. Efficacy and tolerability of controlled release paroxetine in the treatment of panic disorder. J Clin Psychiatry 2005; 66 (1): 3440

40. Ströhle A, Jahn H, Montkowski A et al. Central and peripheral administration of atriopeptin is anxiolytic in rats. Neuroendocrinology 1997; 65: 210-215

41. Simon NM, Worthington J], Doyle AC, Hoge EA, Kinrys G, Fischmann D, Link N, Pollack MH. An open-label study of levetiracetam for the treatment of social anxiety disorder. J Clin Psychiatry 2004; 65 (9): 12191222

42. Spooren W, Gasparini F. mGlu5 receptor antagonists: a novel class of anxiolytics? Drug News Perspect 2004; 17(4): 251-257 43. Ströhle A, Kellner M, Holsboer $F$ et al. Anxiolytic activity of atrial natriuretic peptide in patients with panic disorder. Am J
Psychiatry 2001; 158 (9): 1514-1514

44. Tatarczynska E, Klodzinska A, Stachowicz K, Chojnacka-Wojcik E. Effects of a selective $5-\mathrm{HT} 1 \mathrm{~b}$ receptor agonist and antagonists in animal models of anxiety and depression. Behav Pharmacol 2004; 15 (8): 523-534

45. Van Megen HJ, Westenberg HG, Den Boer JA et al. The cholecystokinin-B receptor antagonist $\mathrm{Cl}-988$ failed to affect CCK-4 induced symptoms in panic disorder patients. Psychopharmacology Berl 11997; 29: 243 248

46. Van Geffen EC Hugtenburg JG, Heerdink $E R$, van Hulten RP, Egberts AC. Discontinuation symptoms in users of selective serotonin reuptake inhibitors in clinical practice: tapering versus abrupt discontinuation. Eur J Clin Pharmacol 2005 (in press)

47. Van Vliet IM, Westenberg HG, Den Boer JA. Effects of the $5 \mathrm{HT} 1 \mathrm{~A}$ receptor agonist flesonoxan in panic disorder. Psychopharmacology Berl 1996; 127: 174-180

48. Vendruscolo LF, Takahashi RN, Bruske $G R$, Ramos A. Evaluation of the anxiolyticlike effect of NKP608, a NK1-receptor antagonist, in two rat strains that differ in anxiety-related behaviors. Psychopharmacology (Bull) 2003; 170 (3): 287-293

49. Versiani M, Cassano G, Perugi G, Bene- detti A, Mastalli L, Nardi A, Savino M. Reboxetine, a selective norepinephrine reuptake inhibitor, is an effective and well-tolerated treatment for panic disorder. J Clin Psychiatry $2002 ; 63(1): 31-7$

50. Wedekind D, Bandelow B. Die Alpha-2Delta Untereinheit des spannungsabhängigen Kalziumkanals. Ein neues pharmakologisches Ziel in der Psychiatrie und Neurologie. Nervenarzt (im Druck)

51. Wolkowitz OM, Reus VI, Chan T et al. Antiglucocorticoid treatment of depression: double-blindketokonazole. Biol Psychiatry 1999; 45 (8): 1070-1074

52. Worthington JJ 3rd, Kinrys G, Wygant LE, Pollack MH. Aripiprazole as an augmentor of selective serotonin reuptake inhibitors in depression and anxiety disorder patients. Int Clin Psychopharmacol 2005; 20 (1): 9-11

\section{Korrespondenzadresse:}

Dr. med. Dirk Wedekind MSc und Prof. Dr. med. Dipl.-Psych. Borwin Bandelow Klinik für Psychiatrie und Psychotherapie der Georg-August-Universität Göttingen Von-Siebold-Straße 5

37075 Göttingen

dwedeki1@gwdg.de 